In Crescendo, 2020; 11(2): 173-186

Fecha de recepción: 30/06/2019

Fecha de aceptación: 18/04/2020

\title{
PROPUESTA DIDÁCTICA PARA EL FORTALECIMIENTO DE LA EDUCACIÓN INCLUSIVA
}

\author{
DIDACTIC PROPOSAL FOR THE STRENGTHENING \\ OF INCLUSIVE EDUCATION
}

Tulia Bibiana Ramón-Jaimes ${ }^{1}$

\section{RESUMEN}

La presente investigación estuvo enfocada al fortalecimiento de la educación inclusiva en los estudiantes de tercer grado del Colegio Camilo Daza de San José de Cúcuta, para lo cual se tomó como fundamento pedagógico la fábula. La revisión y construcción teórica del marco de referencia, permitió reafirmar que para lograr escuelas inclusivas se precisan cambios en los procesos administrativos, curriculares, evaluativos, de seguimiento y de control. Se utilizó un tipo de investigación cualitativo, con el método Acción - Participación, partiendo de una etapa de diagnóstico, en la cual se logró establecer que los estudiantes en mención presentaban algún grado de exclusión hacia sus compañeros en aspectos como género, aspecto físico y tendencias sexuales. Con base en ello, se diseñó una estrategia didáctica basada en la fábula. El desarrollo de la estrategia buscó concientizar la importancia de mejorar las relaciones personales al interior del aula y reconocer la igualdad entre pares. La implementación de la estrategia fue aceptada por los estudiantes, favoreciendo el trabajo en grupo y haciendo uso de las Tecnologías de la Información y la Comunicación (TIC) como recurso didáctico. Para finalizar, se procedió a evaluar la efectividad de la estrategia, de lo cual es preciso mencionar que, en su mayoría, los estudiantes cambiaron, su actitud frente a compañeros y docentes; se integraron por medio de trabajos en grupo y lograron altos índices de tolerancia y respeto por la opinión, creencias y tendencias sexuales y sociales de sus compañeros.

PALABRAS CLAVE: Educación inclusiva, fábula, exclusión relaciones interpersonales, Tecnologías de la Información y la Comunicación (TIC).

1 Maestra en Educación. Docente del Colegio Camilo Daza. Cúcuta. Colombia. vivianaramonjs@gmail.com, https://orcid.org/0000-0002-9075-5006 


\begin{abstract}
This research was focused on strengthening inclusive education in third-grade students at the Camilo Daza School in San José de Cúcuta, for which the fable was taken as a pedagogical foundation. The theoretical review and construction of the reference framework will reaffirm that to achieve inclusive schools, changes are required in the administrative, curricular, evaluative, monitoring and control processes. A qualitative type of research was used, with the Action-Participation method, starting from a diagnosis stage, in which it was established that the students in question presented some degree of exclusion towards their classmates in aspects such as gender, physical appearance and tendencies sexual. Based on this, a didactic strategy based on the fable was designed. The development of the strategy sought to raise awareness of the importance of improving personal relationships within the classroom and to recognize equality among peers. The implementation of the strategy was accepted by the students, favoring group work and making use of Information and Communication Technologies (ICT) as a didactic resource. Finally, we proceeded to evaluate the effectiveness of the strategy, of which it is necessary to mention that, for the most part, the students changed their attitude towards classmates and teachers; they were integrated through group work and achieved high levels of tolerance and respect for the opinion, beliefs and sexual and social tendencies of their peers.
\end{abstract}

KEY WORDS: Inclusive Education, fable, exclusion of interpersonal relations, Information and Communication Technologies (ICT).

\title{
INTRODUCCIÓN
}

La educación inclusiva es definida por la UNESCO, (2003 y 2009) citada por (Opertti, 2011), como:

"Un proceso de abordaje y respuesta a la diversidad de necesidades de todos los estudiantes a través del aumento de la participación en el aprendizaje, las culturas y las comunidades, y la reducción de la exclusión dentro y desde la educación".

En tal sentido, se debe tener en cuenta que dentro de los factores que afectan de forma negativa a los estudiantes están los niveles de exclusión en los que en ocasiones se hallan inmersos.

Se debe anotar que el derecho a la educación en igualdad de condiciones, contribuye al desarrollo y formación de ciudadanos, a fomentar respeto y valoración por la cultura, las diferencias sociales e individuales, a la convivencia pacífica y activa y la construcción de su propia identidad y la del contexto donde se desarrolla entre otras. En tal sentido, el Ministerio de Educación Nacional 
(2013), expresa que la educación inclusiva está relacionada con la capacidad de potenciar y valorar la diversidad, además de promover el respeto de ser diferente y garantizar la participación de la comunidad dentro de una estructura intercultural en los procesos educativos.

Con base en estos fundamentos es procedente mencionar que en las aulas se observan fricciones permanentes entre los escolares, docentes, directivos y padres de familia; aspectos que llevan a la desmotivación, la inseguridad, la desconfianza, la incertidumbre, además de los ambientes que perjudican el sano entendimiento de las particularidades y de las condiciones colectivas.

En este sentido, se debe mencionar que en las aulas se observan problemas de exclusión entre los mismos estudiantes, ante la indiferencia y actitudes negativas de profesores y adultos, para enfrentar las causas de los comportamientos de esta; problema que es sometido a la presente investigación con el fin de ser revisada teórica, práctica y metodológicamente, para así acercar las posibles soluciones que dentro del aula de clase pueden darse.

En la primera etapa, fruto del estudio teórico y práctico, se diagnostican las causas de la exclusión y así mismo se analizan las posibles soluciones a esta dificultad, teniendo en cuenta que son escolares en las edades de 8 a 9 años con condiciones cognitivas, de pensamiento y conducción de valores, promoción de capacidades, habilidades, hacia desempeños saludables en la convivencia y el rendimiento académico en la permanecía dentro del sistema educativo. La fundamentación teórica de las características propias de los escolares de estas edades permitió pensar en una estrategia didáctica en la que utilizando la imaginación creadora, creativa, motivadora, como lo es el género literario la fábula permite promover la reflexión, la autocrítica y la apertura al cambio de actitud.

\section{METODOLOGÍA}

La investigación se enmarca dentro del tipo de investigación cualitativa, el cual según (Hernández, Fernández, \& Baptista, 2010), se enfoca en comprender los fenómenos, desde la perspectiva de los participantes en un ambiente natural. El método utilizado fue Investigación - Acción (I-A), cuya finalidad, de acuerdo con, (Hernández, Fernández, \& Baptista, 2014), es comprender y resolver problemáticas específicas de una colectividad vinculadas a un ambiente (grupo, programa, organización o comunidad). Este método se centra en aportar información 
que guíe la toma de decisiones para proyectos, procesos y reformas estructurales, pretendiendo propiciar el cambio social, transformar la realidad (social, educativa, económica, administrativa, etc.) y que las personas tomen conciencia de su papel en ese proceso de transformación.

\section{Diseño metodológico}

La metodología a seguir se presenta en diversas fases, las cuales permiten una perfecta planeación tanto del trabajo escrito como el de campo:

\section{Fase inicial}

Consulta bibliográfica en libros, revistas, artículos y sitios web; útiles en la construcción de los marcos de referencia y metodología

Diseño de instrumentos para la recolección de la información.

\section{Fase de trabajo de campo}

Entrega de instrumentos de recolección de información a expertos para su validación.

Modificación y ajuste de los instrumentos de recolección de información, según criterio de expertos.

Aplicación de los instrumentos de recolección de la información.

\section{Fase analítica}

Tabulación, graficación y análisis cuantitativo y cualitativo de la información e identificación de las diversas formas de exclusión académica que se presentan en los estudiantes objeto de la investigación.

\section{Fase de elaboración de las estrategias pedagógicas}

Diseño de la estrategia didáctica. Para este caso, se diseñaron guías didácticas teniendo en cuenta aspectos tales como el trabajo individual y en grupo, relación con el medio ambiente, el uso de las TIC y las competencias ciudadanas.

\section{Fase de ejecución}

Aplicación de las estrategias diseñadas a los estudiantes de grado tercero del Colegio Camilo Daza de San José de Cúcuta. 


\section{Fase de evaluación}

Evaluación cualitativa de la efectividad de la estrategia aplicada.

\section{Fase informativa}

Socialización de los resultados del proyecto con la comunidad académica del Colegio Camilo Daza de San José de Cúcuta.

Presentación de ponencia.

Sustentación final del proyecto.

\section{Técnicas e instrumentos de recolección de información}

La recolección de la información se realizó por medio de la observación directa a estudiantes, observación directa a la Institución Educativa y entrevista a docentes. Los instrumentos en mención se describen a continuación:

\section{Instrumento $N^{0} 1$}

Formato de observación directa a los estudiantes de tercer grado del Colegio Camilo Daza de San José de Cúcuta a estudiantes con el fin de identificar patrones de comportamiento ante diversas situaciones de exclusión en la cotidianidad escolar. El mencionado instrumento fue también aplicado al finalizar las actividades, siendo tomada como la evaluación final para determinar la efectividad de la estrategia.

\section{Instrumento № 2}

Formato de observación directa a la Institución Educativa, cuyo objetivo fue determinar la presencia de normas y acuerdos en el manual de convivencia con los que cuenta el Colegio Camilo Daza de San José de Cúcuta para atender situaciones de exclusión.

\section{Instrumento № 3}

Formato de entrevista a docentes con el fin de conocer su opinión acerca de aspectos específicos de la educación inclusiva.

\section{Informantes clave}

Estudiantes de básica primaria del Colegio Camilo Daza de San José de Cúcuta.

Docentes de básica primaria del Colegio Camilo Daza de San José de Cúcuta. 


\section{RESULTADOS}

Se presentan a continuación los resultados obtenidos en la presente investigación, lo cual se hace de acuerdo con el planteamiento de cada uno de los objetivos específicos.

\section{Resultados primer objetivo específico}

Para dar respuesta al primer objetivo específico, es decir, "Identificar las diversas formas de exclusión académica que se presentan en los estudiantes de básica primaria del Colegio Camilo Daza de San José de Cúcuta”, se aplicaron tres instrumentos para recolección de información, los cuales fueron categorizados para su mejor interpretación. Dicha información se relaciona en las tablas 1,2 y 3 .

\section{Resultados segundo objetivo específico}

En lo referente al segundo objetivo específico, es decir, "Diseñar una estrategia didáctica basada en La fábula para potenciar la educación inclusiva en los estudiantes mencionados", se presenta a continuación las generalidades de esta.

\section{Estrategia didáctica}

\section{Presentación}

La estrategia didáctica se enfoca en mejorar los aspectos de convivencia y tolerancia al interior del colegio Camilo Daza de Cúcuta, tomando como base el subgénero narrativo de la fábula, toda vez que este tipo de narración presenta en su estructura general una moraleja o enseñanza, la cual marca las pautas para el comportamiento adecuado tanto en el ámbito educativo como familiar y social.

\section{Justificación}

$\mathrm{Al}$ interior de las aulas del colegio Camilo Daza de Cúcuta, se presentan algunos casos de intolerancia y enemistad, generados, en su mayoría por dificultad de aceptar al otro con sus virtudes y defectos, lo cual se configura, en términos generales, como un tipo de exclusión, que a mediano o largo plazo puede derivar 
en el ausentismo y, en muchas ocasiones, la deserción escolar, afectando de esta manera el rendimiento académico y la permanencia en el sistema educativo. Con la presente estrategia se pretende fortalecer los vínculos de amistad, la camaradería la y tolerancia, con el ánimo de crear ambientes sanos de convivencia y de amistad al interior de las aulas, los cuales se vean reflejados en el comportamiento de los estudiantes dentro y fuera de ellas.

\section{Objetivos}

Fortalecer la sana convivencia de los estudiantes del colegio Camilo Daza de Cúcuta, dentro y fuera del aula.

Reconocer la fábula como elemento que permite la reflexión, mejorando los estándares de comportamiento de sus lectores.

\section{Metodología}

Las guías didácticas de la propuesta están diseñadas con una estructura estándar, teniendo en cuenta aspectos tales como:

Título, Imagen de ambientación, Presentación, Justificación, Objetivos, Metodología del trabajo, tanto del docente como del estudiante; Fábula, Preguntas, Trabajo en equipo, utilización de las TIC y Socialización de la actividad.

\section{Resultados cuarto objetivo específico}

Para dar respuesta al planteamiento del cuarto objetivo específico, es decir, "Evaluar la efectividad de la estrategia didáctica aplicada a los estudiantes objeto de la investigación", se presenta en la tabla 4 la aplicación del instrumento de observación directa a los estudiantes, el cual fue aplicado una vez desarrolladas las guías didácticas. 
Tabla 1

INSTRUMENTO NNo 1 OBSERVACIÓN DIRECTA NO PARTICIPANTE A ESTUDIANTES

\begin{tabular}{|c|c|c|}
\hline Categorías & Subcategorías & Análisis \\
\hline \multirow{5}{*}{$\begin{array}{l}\text { Relación } \\
\text { entre } \\
\text { estudiantes }\end{array}$} & $\begin{array}{l}\text { Trabajos en } \\
\text { grupo }\end{array}$ & \multirow{2}{*}{$\begin{array}{l}\text { La relación entre estudiantes se establece básicamente desde los } \\
\text { trabajos que desarrollan en las diferentes clases, allí se percibe que } \\
\text { algunos estudiantes son muy dados a imponer su criterio y no aceptan la } \\
\text { opinión de los demás. }\end{array}$} \\
\hline & Toma de & \\
\hline & $\begin{array}{l}\text { decisiones } \\
\text { en grupo }\end{array}$ & \multirow{2}{*}{$\begin{array}{l}\text { Por otra parte, se percibe que algunos estudiantes muestran desagrado } \\
\text { cuando tienen que desarrollar trabajo en grupo y prefieren hacerlos de } \\
\text { manera individual, lo cual afecta la relación con sus pares. }\end{array}$} \\
\hline & Relación en & \\
\hline & $\begin{array}{l}\text { diferentes } \\
\text { actividades }\end{array}$ & $\begin{array}{l}\text { En actividades culturales, deportivas y sociales (recreo), se percibe que } \\
\text { existen grupos muy cerrados y no incluyen a algunos compañeros con } \\
\text { los que no tienen confianza o porque no comparten algunos aspectos de } \\
\text { su personalidad. }\end{array}$ \\
\hline
\end{tabular}

\begin{tabular}{ll} 
& \multicolumn{1}{c}{$\begin{array}{l}\text { Actividades } \\
\text { académicas }\end{array}$} \\
\cline { 2 - 2 } Exclusión & $\begin{array}{l}\text { Actividades } \\
\text { lúdicas }\end{array}$ \\
escolar por & \\
\cline { 2 - 2 } genero & $\begin{array}{l}\text { Actividades } \\
\text { recreativas }\end{array}$
\end{tabular}

La mayoría de actividades académicas, lúdicas, recreativas y sociales son realizadas por grupos específicos por género, es decir, hombres con hombres y mujeres con mujeres, lo cual impide en gran medida que exista una buena relación de amistad y compañerismo entre pares de distinto género.

En este sentido, es preciso anotar que el desempeño académico y permanencia en el sistema educativo de los estudiantes depende en gran medida de la calidad de la relación que tienen los estudiantes con sus compañeros, máxime si no se tiene en cuenta características de género.

\begin{tabular}{|c|c|c|}
\hline \multirow{4}{*}{ Actitud } & \multirow{2}{*}{$\begin{array}{l}\text { Actitud de } \\
\text { los } \\
\text { estudiantes } \\
\text { en situación } \\
\text { de exclusión } \\
\text { con sus } \\
\text { compañeros. }\end{array}$} & $\begin{array}{l}\text { Se percibe que algunos estudiantes tienen actitudes inadecuadas con } \\
\text { sus compañeros, tales como burlas, críticas y segregación de grupos ya } \\
\text { conformados. }\end{array}$ \\
\hline & & $\begin{array}{l}\text { De acuerdo con lo anterior, se infiere que, en su mayoría, las peleas, } \\
\text { desacuerdos y malos entendidos que se presentan, son consecuencia } \\
\text { de las mencionadas actitudes, las cuales dan visos de intolerancia, } \\
\text { irrespeto y enemistad. }\end{array}$ \\
\hline & \multirow{2}{*}{$\begin{array}{l}\text { Actitud de } \\
\text { los } \\
\text { estudiantes } \\
\text { en situación } \\
\text { de exclusión } \\
\text { con los } \\
\text { docentes. }\end{array}$} & $\begin{array}{l}\text { Respecto a la actitud con los docentes, se puede afirmar que, en su } \\
\text { mayoría, los estudiantes son respetuosos y acatan las indicaciones } \\
\text { dadas de forma oportuna. }\end{array}$ \\
\hline & & $\begin{array}{l}\text { Cabe anotar que se percibe cierto cambio de actitud de los estudiantes } \\
\text { cuando están siendo observados o supervisados por la docente, caso } \\
\text { contrario cuando esto no sucede, lo cual indica que no hay conciencia total } \\
\text { del comportamiento que deben tener en todo momento y en todo lugar. }\end{array}$ \\
\hline
\end{tabular}

Fuente: Propia 
Tabla 2

\section{INSTRUMENTO № 2 OBSERVACIÓN DIRECTAA LA INSTITUCIÓN EDUCATIVA}

\begin{tabular}{|c|c|c|}
\hline Categorías & Subcategorías & Análisis \\
\hline & $\begin{array}{l}\text { Manual de } \\
\text { convivencia }\end{array}$ & \multirow{2}{*}{$\begin{array}{l}\text { La Institución Educativa cuenta con un Manual de Convivencia, el cual } \\
\text { incluye los parámetros de comportamiento de los estudiantes, además } \\
\text { de los protocolos que se ha de seguir en los diversos procedimientos } \\
\text { disciplinarios. }\end{array}$} \\
\hline & & \\
\hline $\begin{array}{l}\text { Convivencia } \\
\text { escolar }\end{array}$ & $\begin{array}{l}\text { de la } \\
\text { comunidad } \\
\text { educativa en la } \\
\text { creación del } \\
\text { Manual de } \\
\text { Convivencia }\end{array}$ & $\begin{array}{l}\text { El manual de convivencia es autoría del Consejo Superior, donde cada } \\
\text { uno de los estamentos de la Institución Educativa tiene representación, } \\
\text { sin embargo, la apreciación general es no sentirse debidamente } \\
\text { representados en sus expectativas, deseos y necesidades, lo cual se } \\
\text { traduce en un sentimiento de no hacer parte de la construcción del } \\
\text { mencionado manual. }\end{array}$ \\
\hline
\end{tabular}

\begin{tabular}{|c|c|c|}
\hline \multirow{4}{*}{$\begin{array}{l}\text { Normas y } \\
\text { acuerdos }\end{array}$} & $\begin{array}{l}\text { Actualización } \\
\text { del manual de } \\
\text { convivencia. }\end{array}$ & \multirow{2}{*}{$\begin{array}{l}\text { El Manual de Convivencia de la Institución Educativa, a pesar de que es } \\
\text { revisado y actualizado con alguna regularidad, presenta desactualiza- } \\
\text { ción en aspectos legales, así como de comportamiento y compromisos } \\
\text { por parte de los estudiantes. } \\
\text { En este mismo sentido, se debe mencionar que en su mayoría, los que } \\
\text { participan de la actualización son los profesores, dejando de lado al resto } \\
\text { de la comunidad educativa, y por ende muchos aspectos de gran rele- } \\
\text { vancia. }\end{array}$} \\
\hline & \multirow{2}{*}{$\begin{array}{l}\text { Participación } \\
\text { de la } \\
\text { comunidad } \\
\text { educativa en la } \\
\text { actualización } \\
\text { del manual de } \\
\text { convivencia. }\end{array}$} & \\
\hline & & \multirow{2}{*}{$\begin{array}{l}\text { Referente a la presencia de elementos que favorezcan la inclusión, el } \\
\text { Manual de Convivencia presenta algunas falencias, toda vez que no es } \\
\text { explícito en la manera como los docentes y estudiantes deben asumir } \\
\text { actitudes y responsabilidades que garanticen el respeto hacia las creen- } \\
\text { cias, raza, tendencias sexuales y posición social, las cuales inciden } \\
\text { directamente sobre el desempeño académico y la permanencia en el } \\
\text { sistema educativo. }\end{array}$} \\
\hline & $\begin{array}{l}\text { Presencia en } \\
\text { el manual de } \\
\text { convivencia de } \\
\text { elementos que } \\
\text { favorecen la } \\
\text { inclusión }\end{array}$ & \\
\hline
\end{tabular}

Fuente: Propia 


\section{Tabla 3}

\section{INSTRUMENTO N 3 ENTREVISTAA DOCENTES}

\begin{tabular}{|c|c|c|}
\hline Categorías & Subcategorías & Análisis \\
\hline \multirow{5}{*}{$\begin{array}{l}\text { Formación } \\
\text { del maestro }\end{array}$} & \multirow{2}{*}{$\begin{array}{l}\text { Formación } \\
\text { académica } \\
\text { relacionada con } \\
\text { la atención de } \\
\text { estudiantes en } \\
\text { situación de } \\
\text { exclusión }\end{array}$} & $\begin{array}{l}\text { La totalidad de los docentes entrevistados manifestaron no tener formación } \\
\text { académica que les permita atender debidamente a los estudiantes que. por } \\
\text { diversas razones se encuentran en situación de exclusión, lo cual va en } \\
\text { contravía de los fines de la educación y de la permanencia misma de estos } \\
\text { estudiantes en el sistema. }\end{array}$ \\
\hline & & $\begin{array}{l}\text { De la misma manera, la totalidad de los docentes entrevistados expresaron } \\
\text { que no reciben capacitaciones ni actualizaciones para atender debidamente }\end{array}$ \\
\hline & & \\
\hline & $\begin{array}{l}\text { y actualizaciones } \\
\text { para atender } \\
\text { situaciones de } \\
\text { exclusión en el } \\
\text { aula }\end{array}$ & $\begin{array}{l}\text { Así mismo, la mayoría de los docentes enunciaron no conocer la Ley } 1620 \text { de } \\
2013 \text {, la cual crea y regula el Sistema Nacional de Convivencia Escolar y } \\
\text { Formación para el Ejercicio de los Derechos Humanos, la Educación para la } \\
\text { Sexualidad y la Prevención y Mitigación de la Violencia Escolar; aspecto que, } \\
\text { en términos pedagógicos, no favorece en medida alguna la inclusión al } \\
\text { interior de las aulas. }\end{array}$ \\
\hline & $\begin{array}{l}\text { Conocimiento de } \\
\text { la legislación } \\
\text { vigente }\end{array}$ & $\begin{array}{l}\text { Lo anterior supone que los docentes deben hacer frente a dichas situaciones } \\
\text { de acuerdo con su instinto, pues no cuentan con los principios teóricos que } \\
\text { les permitan tomar la decisión correcta en cada una de las situaciones. }\end{array}$ \\
\hline
\end{tabular}

Promoción de actividades que favorecen la inclusión.

Estrategias utilizadas para generar inclusión.

Aportes que

Procesos generan pedagógicos
Dentro de las actividades realizadas en el aula que favorecen la inclusión, los docentes mencionan los trabajos en grupo, además del desarrollo actividades lúdicas y recreativas.

De la misma manera, mencionan el trabajo desarrollado con el proyecto de convivencia y paz, el cual está enfocado a mejorar las relaciones interpersonales entre los estudiantes y entre estos y los docentes.

En el mismo sentido, los docentes mencionan la promoción del diálogo, exposiciones cuyo tema central son los valores el respeto y la tolerancia, incluyendo estos temas, además, de forma transversal en cada una de las asignaturas.

Por último, los docentes mencionan que en ocasiones deben hacer llamados de atención a los estudiantes y sugerirles el cambio de actitud frente a situaciones donde no se evidencie la tolerancia y el respeto hacia los compañeros.

En lo referente a los aportes que hacen los docentes para generar inclusión en la Institución Educativa, mencionan que los padres de familia también deben asumir con responsabilidad el mencionado tema y guiar a sus hijos hacia la toma de decisiones acertadas.

De la misma manera, mencionan la importancia de la formación y capacitación que se debe dar a los docentes en temas de inclusión, con el fin de manejar debidamente cada situación en particular.

Por otra parte, mencionan que es necesario mejorar la planta física y contar con los recursos didácticos necesarios para la formación de los estudiantes, en aras de manejar correctamente el tema.

Fuente: Propia 
Tabla 4

INSTRUMENTO NN 4 CATEGORÍAS Y SUBCATEGORÍAS CUARTO OBJETIVO

\begin{tabular}{|c|c|c|}
\hline Categorías & Subcategorías & Análisis \\
\hline \multirow{3}{*}{$\begin{array}{l}\text { Relación } \\
\text { entre } \\
\text { estudiantes }\end{array}$} & $\begin{array}{l}\text { Trabajos en } \\
\text { grupo }\end{array}$ & \multirow{3}{*}{$\begin{array}{l}\text { Luego de aplicadas las actividades y del énfasis aplicado en cada una de } \\
\text { ellas respecto a la importancia del trabajo en grupo y el respeto por la } \\
\text { opinión y la personalidad de los demás, se percibe que la mayoría de } \\
\text { estudiantes comprendieron el mensaje y han logrado establecer mejores } \\
\text { relaciones con sus compañeros, lo cual se evidencia en el uso de la palabra } \\
\text { y en los acuerdos a los que llegan sin necesidad de entrar en discusiones } \\
\text { vanas. } \\
\text { En el mismo sentido, se percibe que, en su mayoría, los estudiantes son } \\
\text { más tolerantes y menos excluyentes con sus compañeros, mayormente en } \\
\text { aspectos como el género, el aspecto físico y la forma de pensar. Dicha } \\
\text { situación, ha mejorado notoriamente el clima de convivencia al interior del } \\
\text { aula. }\end{array}$} \\
\hline & $\begin{array}{l}\text { Toma de } \\
\text { decisiones en } \\
\text { grupo }\end{array}$ & \\
\hline & $\begin{array}{l}\text { Relación en } \\
\text { diferentes } \\
\text { actividades }\end{array}$ & \\
\hline
\end{tabular}

\begin{tabular}{lll}
\hline & $\begin{array}{l}\text { Actividades } \\
\text { académicas }\end{array}$ & $\begin{array}{l}\text { Las actividades académicas, lúdicas y recreativas desarrolladas por la } \\
\text { mayoría de los estudiantes, son ahora más dinámicas y cuentan con la } \\
\text { participación de todos los estudiantes, logrando de esta manera fortalecer }\end{array}$ \\
\cline { 2 - 3 } $\begin{array}{l}\text { Exclusión } \\
\text { escolar por }\end{array}$ & $\begin{array}{l}\text { Actividades } \\
\text { los vínculos de amistad y camaradería, lo cual a mediano y largo plazo ha } \\
\text { genero }\end{array}$ & $\begin{array}{l}\text { de verse reflejado en el rendimiento académico y en los índices de } \\
\text { permanencia en el sistema educativo. }\end{array}$ \\
\cline { 2 - 3 } & $\begin{array}{l}\text { Actividades } \\
\text { recreativas }\end{array}$ & $\begin{array}{l}\text { Cabe destacar que las actividades mencionadas han logrado salir de la } \\
\text { monotonía, toda vez que al tener en cuenta la opinión y la participación de la } \\
\text { mayoría de estudiantes, se hacen más entretenidas, fluidas y divertidas. }\end{array}$
\end{tabular}

Actitud de los estudiantes en situación de exclusión con sus compañeros.

Actitud Actitud de los estudiantes en situación de exclusión con los docentes.
En términos generales, se percibe que las moralejas dejadas por las fábulas trabajadas, han surtido un efecto positivo en la mayoría de estudiantes, toda vez que su actitud ha cambiado y se sienten ahora parte del grupo, pues allí no se les discrimina por su condición sexual, o social, ni mucho menos por sus creencias o por su aspecto físico.

Se debe destacar también, que la actitud de los estudiantes con los docentes ha sido muy positiva y de receptividad, lo cual ha mejorado de manera ostensible la relación entre los unos y los otros.

Con base en lo anterior, se puede afirmar que la estrategia implementada cumplió con los objetivos establecidos, pues los estudiantes lograron entender que, ante la sociedad, todos somos iguales, y que las diferencias sociales, de raza, de religión y de tendencias religiosas no son impedimentos para llevar una relación sana de compañerismo y amistad.

Fuente: Propia 


\section{CONCLUSIONES}

Considerando que los objetivos planteados para la presente investigación son la base para establecer las conclusiones, se presentan a continuación los aspectos más relevantes en cada uno de ellos:

De acuerdo con lo anterior, se puede afirmar que se identificaron diversas formas de exclusión académica que se presentan en los estudiantes de básica primaria del Colegio Camilo Daza de San José de Cúcuta, dentro de las cuales se destacan:

Exclusión de género, pues los niños prefieren desarrollar actividades académicas, lúdicas y recreativas con pares del mismo sexo.

Exclusión por aspecto físico, lo cual se evidencia en las burlas que hacen los estudiantes a sus compañeros por ser gordos o delgados, altos o bajitos, etc.

Exclusión socioeconómica, evidenciada esta en apartar de grupos de trabajo a los compañeros más pobres o mal vestidos.

Exclusión religiosa, la cual se percibe en las incompatibilidades de criterios cuando no coinciden con las creencias y planteamientos del credo de uno con el otro.

Con base en los resultados de la aplicación del instrumento anterior, se procedió a dar respuesta al segundo objetivo específico, para lo cual se procedió a diseñar una estrategia didáctica basada en la Fábula para potenciar la educación inclusiva en los estudiantes mencionados. Es preciso anotar que se eligió la fábula por ser un subgénero del género narrativo que deja una enseñanza por medio de una historia corta, lo cual resultó muy funcional para los estudiantes en cuestión.

Cabe anotar que cada una de las guías didácticas contó con actividades individuales y grupales, incluyendo las TIC y su respectiva socialización; aspectos que favorecieron en gran medida su desarrollo, y logrando así un verdadero aprendizaje significativo.

Respecto al tercer objetivo específico, se debe mencionar que se aplicó la estrategia diseñada a los estudiantes de básica primaria del Colegio Camilo Daza de San José de Cúcuta, de la cual se pueden mencionar que los estudiantes:

Desarrollaron con interés cada una de las actividades.

Respondieron adecuadamente cada una de las interrogantes planteadas.

Incorporaron las TIC a su proceso educativo. 
Respecto al cuarto y último objetivo, se evaluó la efectividad de la estrategia didáctica aplicada de lo cual se puede decir que los estudiantes:

Se integraron a grupos de trabajo sin distingo de género, raza, creencias o nivel socioeconómico.

Hicieron acuerdos, pactos y arreglos para presentar sus trabajos y actividades.

Comprendieron la importancia del respeto y la tolerancia en aras de mejorar las relaciones interpersonales.

Fortalecieron su capacidad de comprensión y argumentación respecto a los textos abordados.

Mejoraron el clima de amistad y camaradería al interior del aula.

\section{RECOMENDACIONES}

Con base en los resultados de la investigación realizada, se recomienda a los docentes:

Trabajar la inclusión de forma transversal en todas las áreas, y no dejar el tema para ser trabajado de forma exclusiva en algunas ocasiones.

Utilizar ejemplos prácticos y vivenciales para abordar con los estudiantes el tema de la inclusión de forma significativa.

Brindar las condiciones académicas, didácticas y de infraestructura a los estudiantes para que hagan efectivos los criterios de inclusión en el aula.

Dar trato sin discriminación alguna a los estudiantes, con el fin de servir como ejemplo para ellos mismos.

Utilizar el cuento, la fábula y demás subgéneros de la literatura infantil como elemento para fortalecer la inclusión.

\section{De la misma manera, se sugiere a los estudiantes:}

Reconocer en sus compañeros su imagen propia, respetándola y valorándola como persona importante en el ámbito social y académico.

Utilizar todas ventajas e información que brinda la literatura para mejorar las relaciones con sus compañeros, docentes, familiares y amigos.

Acatar las observaciones dadas por los docentes respecto a la integración con sus compañeros y al respeto por sus creencias y tendencias sociales, religiosas y sexuales. 


\section{REFERENCIAS BIBLIOGRÁFICAS}

Abrate, Raquel, Pochulu, M., \& Vargas, J. (2006). Errores y dificultades en matemática Análisis de causas y sugerencias de trabajo. Recuperado el 20 de Noviembre de 2017, de http://unvm.galeon.com/ Libro1.pdf.

Balcazar, P., González, N., \& Gurrola, G. M. (2013). Investigación Cualitativa. Recuperado el 11 de Agosto de 2018, de http://repositorio.minedu.gob.pe/handle/123456789/4641

Colmenares, A. (Agosto de 2008). La Investigación-Acción. Una herramienta metodológica heurística para la comprensión y transformación de realidades y prácticas socio-educativa. Recuperado el 12 de Septiembre de 2018, de http://www.redalyc.org/pdf/761/76111892006.pdf

Congreso de la República. (8 de Noviembre de 2006). Ley 1098. Recuperado el 30 de Agosto de 2018, de https://www.icbf.gov.co/cargues/avance/docs/ley_1098_2006.htm

Crosso, C. (26 de Agosto de 2014). El derecho a la educación de personas con discapacidad. Impulsando el concepto de educación inclusiva. Recuperado el 2 de Agosto de 2018, de http://www.reposi toriocdpd.net:8080/handle/123456789/413

DANE. (2005). Cálculos de Economía y Desarrollo. Recuperado el 24 de Agosto de 2018

Dominguez, L. (2009). poyo familiar y desempeño en lengua escrita en escolares de quinto grado de primaria. México.

Donato, R., Kurlat, M., Padín, C., \& Rusler, V. (2014). Experiencias de inclusión educativa desde laperspectiva de aprender juntos. Recuperado el 2 de Mayo de 2017, de https://www.unicef.org/argentina/ spanish/Inclusion_Educativa.pdf.

Educarchile. (2001). Evaluación Incial o Diagnóstica. Recuperado el 11 de Marzo de 2018, de http://ww2.educarchile.cl/UserFiles/P0001\%5CFile\%5CEvaluaci\%C3\%B3n\%20Inicial.pdf

Elliot, J. (2000). La investigación- acción en educación. Recuperado el 2 de Febrero de 2018, de http://www.terras.edu.ar/biblioteca/37/37ELLIOT-Jhon-Cap-1-y-5.pdf

Fals Borda, O. (1991). Acción y conocimiento: Rompiendo el monopilio con la IAP. Bogotá: Rahman.

Feo, R. J. (2010). Orientaciones básicas para el diseño de estrategias didácticas. UPEL. Caracas, Venezuela: Tendencias Pedagógicas.

Gómez, f. (24 de julio de 2012). Elementos problemáticos en el proceso de enseñanza de las matemáticas en estudiantes vicente abadía. Recuperado el 23 de noviembre de 2017, de www.bdigital.unal.edu.co/7052/1/7810033.2012.pdf

Opertti, R. (2011). La educación inclusiva; Perspectiva internacional y retos del futuro. Recuperado el 1 de Septiembre de 2016, de https://scholar.google.com/scholar?q=tesis+de+LA+EDUCACION+ INCLUSIVA +EN + LA + educacion + superior $\& h l=$ es\&as_sdt $=0 \&$ \&as_vis $=1 \& o i=$ scholart \&sa $=$ $\mathrm{x} \&$ ved $=0$ ahukewjms6fl0otpahwtub4khzp1 bjqqgqmihdaa

Pabón, R., \& Barrantes, R. (Abril de 2012). Las Tensiones de la Educación Inclusiva. Recuperado el 20 de Agosto de 2016, de https://scholar.google.com/scholar?start=10\&q=definicion + de + educacion + inclusiva $+\mathrm{pdf}+$ academico $\& h \mathrm{l}=$ es\&as_sdt $=0,5 \& a s \_v i s=1$

Pimienta, R. (2002). Encuestas probabilísticas vs. no probabilística. Recuperado el 3 de Mayo de 2017, de http://www.redalyc.org/pdf/267/26701313.pdf

Rodríguez, S., Heraíz, N., \& Prieto, M. (2011). Investigación Accion. Recuperado el 6 de Agosto de 2017, de https://www.uam.es/personal_pdi/stmaria/jmurillo/InvestigacionEE/Presentaciones/Curso_10/Inv_ accion_trabajo.pdf

Varela, D. (2014). La relación entre las prácticas educativas de los docentes de educación especial y de educación primaria hacia la adopción de un enfoque inclusivo, una aproximación etnográfica. Recuperado el 2 de Mayo de 2017, de http://cdigital.uv.mx/bitstream/123456789/42148/1/VarelaSaucedoDiana.pdf 